\title{
Recent Advance in Lymph Dynamic Analysis in Lymphatics and Lymph Nodes
}

Fumitaka Ikomi, MD, PhD, Yoshiko Kawai, MD, PhD, and Toshio Ohhashi, MD, PhD

Lymphatics are a unidirectional transport system that carries fluid from the interstitial space and back into the blood stream. Initial lymphatics take up not only fluid but also high-molecular-weight substances, such as plasma proteins and hyaluronan; immune cells, such as lymphocytes, macrophages, and dendritic cells; and colloidal particles, such as carbon particles, bacteria, and tattoo dye. Interstitially injected colloidal particles are known to accumulate in the regional lymph nodes. This phenomenon is applied to find sentinel lymph nodes in cancer patients. Lymph flow rate and composition are influenced by interstitial fluid, lymphatic pump activity, and intra-lymphatic pressure. Lymph composition is changed during its flow downstream. In this review, the main focus is on the mechanisms of lymph formation at the initial lymphatics and lymph transport through the collecting lymphatics and lymph nodes. (*English Translation of J Jpn Coll Angiol, 2008, 48: 113-123.)

Keywords: lymph flow, sentinel lymph node, colloidal particle, drug delivery system (dds), ultrasound contrast medium

\section{INTRODUCTION}

$\mathrm{T}$ he lymphatic system is a vascular system existing in parallel with the blood vessel system, and its primary function is considered to be transport of materials through lymph vessels. The fluid in the lymph vessels is called lymph or lymphatic fluid and is transported from upstream to downstream in the lymphatic system. The lymph vessels fuse with the venous system at their downstream ends (thoracic duct-left venous angle, right lymphatic trunk-right venous angle). Unlike the blood vessel system, which is a closed circulation system, the lymphatic system is a one-way channel originating in the

Department of Physiology, Shinshu University School of Medicine, Matsumoto, Nagano, Japan

Received: April 10, 2012; Accepted: June 15, 2012

Corresponding author: Toshio Ohhashi, MD, PhD. Department of Physiology, Shinshu University School of Medicine, 3-1-1, Asahi, Matsumoto, Nagano 390-8621, Japan

Tel: +81-0263-37-2597, Fax: +81-0263-36-5149

E-mail: ohhashi@shinshu-u.ac.jp

*This article is English Translation of J Jpn Coll Angiol, 2008, 48: 113-123. initial lymphatics and distributed to various tissues of the body. Because of this structural difference, while the blood circulates by the ejection force of the heart, lymph is transported by the lymph vessels themselves working as pumps. For this reason, while blood usually flows from areas with a higher blood pressure to areas with a lower blood pressure, the internal pressure of the lymphatic system is often higher downstream than upstream. ${ }^{1)}$ Moreover, as a characteristic of the lymph circulation, there are lymph nodes in the lymphatic channels, and they function as filters that trap foreign bodies carried by the lymph flow, as transport channels and reaction sites of immune cells patrolling the body, and as primary sites of lymph concentration. ${ }^{2)}$

In the 1990s, VEGF-C and VEGFR-3 (Flt4), a factor that specifically proliferates lymph vessels and its receptor, respectively, were identified. In association, knowledge about the development of the lymphatic system and the relationship of lymphangiogenic factors with lymphogenous metastasis of tumors increased markedly. At the same time, the concept of sentinel lymph node began to be accepted clinically. ${ }^{4}$ The sentinel lymph node theory is the concept that there is no metastasis in 
lymph nodes farther than sentinel lymph nodes if sentinel lymph nodes, or lymph nodes that lymph produced around the primary focus of tumor reaches first, are free of metastasis. According to this theory, identification of sentinel lymph nodes makes possible the determination of the surgical strategy concerning lymph node dissection. ${ }^{5)}$ In these circumstances, much attention has been recently directed to the lymphatic system.

The roles of the lymphatic system includes not only recovering tissue fluid into the blood circulation via the lymph circulation and serving as a route of transport of immune cells but, pathophysiologically, also providing a route of metastasis to tumor. Therefore, knowledge about lymph kinetics is indispensable for understanding the mechanisms of these functions. In this paper, primarily the lymph flow in a normal state is described in connection with the significance of the lymphatic system.

\section{Roles of The Lymphatic System ANd Lympho- KINETICS}

To evaluate the characteristics of the lymph circulation, the significance of the lymphatic system and lymph circulation is discussed serially.

\section{(1) Recovery of plasma components that have escaped} from the blood vascular system into circulating blood Water is exchanged between plasma and tissue fluid via the wall of the microvasculature according to Starling's hypothesis. ${ }^{6)}$ Also, plasma protein leaks out primarily through the venular wall. The amount of albumin recovered daily from tissue spaces by the lymphatic system is nearly half the total amount contained in the circulating blood. Since both the static hydraulic pressure and plasma protein concentration are higher in the blood vessels than in tissue spaces, plasma protein that has leaked into tissue spaces cannot return directly to plasma, because plasma protein causing the colloid osmotic pressure diffuses outward even if water can be transported into the blood vessels due to colloid osmotic pressure differences. Thus, it is necessary to recover plasma protein from tissue spaces by the pumping activity of the lymph vessels. ${ }^{7)}$ For this reason, the lymphatic system is considered to be contributing to the homeostasis of the environment around cells and maintenance of circulating plasma.

\section{(2) Transport of water, electrolytes, and nutrients} absorbed via the digestive tract

The origins of the lymph vessels called central lacte- als are present in the villi of the small intestine and, in digestion and absorption, transport lipid soluble materials particularly as chylomicrons. Moreover, the absorption of lipids is known to be closely related to the gastrointestinal immune system. ${ }^{8)}$

\section{(3) Transport of physiologically active materials such as hormones and enzymes released or secreted from cells}

Fenestrated capillaries with a structure facilitating the transport of macromolecular hormones into plasma, are present in endocrine glands. In these organs, the lymph vessels are also developed, and lymph from the adrenal gland, pancreas, thyroid, kidney, and small intestine contains high concentrations of cortisol, insulin, thyroxin, renin, and alkaline phosphatase. ${ }^{9)}$

(4) Elimination of macromolecular materials and particulate components from tissue spaces

Tattoo dyes that have entered the tissue space have long been known to be accumulated in the regional lymph nodes. ${ }^{10)}$ The lymph vessels take up and transport macromolecules that constitute tissue space such as hyaluronic acid, micro/nanoparticles such as carbon particles, and foreign bodies such as bacteria, and these particles are disposed of or accumulated in the lymph nodes. By these activities, homeostasis of the microenvironment of the tissue space around cells is maintained. Also, utilizing these characteristics of the lymph vessels and lymph nodes, methods to identify sentinel lymph nodes using various colloid particles as tracers have been developed.

(5) Channel of movement and site of reaction of antigens, antibodies, and immune cells

Lymphocytes, which circulate in the blood stream migrate extravascularly from high endothelial venules (HEV) in lymph nodes and recognize antigen-presenting cells flowing in through the afferent lymph vessels. The movements of these immune cells are regulated by various adhesion molecules and chemokines. ${ }^{11)}$

\section{(6) Secretion of physiologically active components}

From lymph vessel endothelial cells, nitric oxide (NO) and prostaglandins are released on stimulation of the lymph vessel lumen by the flow and various drugs. These physiologically active materials released are known to be able to regulate the tension of the accompanying blood vessels. ${ }^{12)}$ In the microcirculation system, the artery and vein, which flow in the opposite 
directions, are considered to exchange materials, heat, and information, ${ }^{13)}$ but the lymph vessel also flows in the opposite direction to the accompanying artery and may be involved in the regulation of the blood flow in a manner dependent on the state of the tissue.

\section{(7) Pathophysiologic significance}

Abnormalities of the lymphatic system induce edema and protein losing enteropathy. Lymphatic edema of the limbs occurs primarily after lymph node dissection or radiation therapy of the axilla or pelvis for breast cancer or uterine cancer. The site that has developed lymph edema becomes vulnerable to phlegmon and eventually presents a state of elephantiasis. Furthermore, there are life-threatening types of edema including brain edema, which induces brain herniation, laryngeal edema/pulmonary edema, which causes respiratory insufficiency, and excessive accumulation of pericardial effusion, which causes cardiac tamponade. Edema of the myocardium also causes impairment of both contraction and dilation. ${ }^{14)}$

In contrast with lymphatic edema caused by the arrest of the lymph flow due to obstruction of the lymph vessels, a marked increase in the lymph flow is observed in edema accompanying congestive heart failure or liver cirrhosis. The lymph flow in the human thoracic duct is about $1 \mathrm{~mL} / \mathrm{min}$ in a physiologic state, but it has been reported to increase 3-12 times in these pathologic conditions. ${ }^{15}$ )

The lymphatic system is also known to be a route of tumor metastasis. To improve the QOL of tumor patients, the accurate identification of the sentinel lymph node and the development of more effective methods for the diagnosis and treatment of lymph node metastasis are urgently needed. For this purpose, DDS targeted to the lymphatic system using colloid particles are being developed.

\section{Flows in the Lymph Vessels}

Lymph is generated as tissue fluid is pumped up. Tissue fluid is free water present in the tissue space. The composition of lymph is known to change during the processes of its production and transport, and it must be noted that it is not the same as tissue fluid while it reflects the properties of tissue fluid.

The part of the lymphatic system that produces lymph in tissues is called the initial lymphatic, and the part that collects lymph from the initial lymphatics and transports it downstream is called the collecting lymphatic. Of the collecting lymphatics, particularly large vessels such as the lumbar trunk, intestinal trunk, right trunk, and tho- racic duct, which gather lymph from various areas, are also called lymphatic trunks. Lymph vessels that carry lymph into lymph nodes are called afferent lymphatics (prenodal lymphatics), and those that drain lymph from lymph nodes are called efferent lymphatics (postnodal lymphatics). ${ }^{16)}$

Unlike the blood vascular system, the lymphatic system has no system that drives the entire circulation system such as the heart. Therefore, the lymphatic system needs special mechanisms for the transport of lymph. The mechanisms primarily of lymph production and transport are described below.

\section{(1) Structure of tissue spaces and properties of tissue fluid}

The tissue space is the gap between cells in each tissue. In this part, highly water-retentive gelatinous materials called proteoglycans fill the spaces in the mesh made of fibrous components such as collagen fibers and elastic fibers (Fig. 1). Cells are also distributed in the mesh of these fibrous components. Also, proteins considered to be involved in the adhesion of these materials such as fibronectin, laminin, tenascin, and vitronectin are also present. Tissue fluid, which is free water in the tissue space, exists as very narrow channels $1 \mathrm{~mm}$ or less in diameter in the spaces between these fibers, gel, and cells. Edema occurs primarily due to an increase in free water as well as swelling of gel. ${ }^{2)}$

The evaluation of the properties of tissue fluid is difficult because of the pattern of its distribution. In measuring the hydrostatic pressure of tissue fluid, methods including the porous capsule implantation, cotton core method, and fine glass pipette insertion have been devised, resulting in the clarification that a hydrostatic pressure of tissue fluid in the skin is maintained at a few mmHg lower than the atmospheric pressure. ${ }^{2)}$

\section{(2) Mechanism of lymph production}

The initial lymphatics located most upstream in the lymphatic system are made of a single layer of endothelial cells and a non-continuous basement membrane. A space with a width of a few micrometers is present between neighboring endothelial cells created by folds of endothelial cells, and tissue fluid is pumped up through this space. Because of such a structure, not only macromolecular materials but also particles in a nanometer order can be taken into the lymph vessels. The folds of endothelial cells serve as valves that prevent regurgitation of tissue fluid that has been taken up 


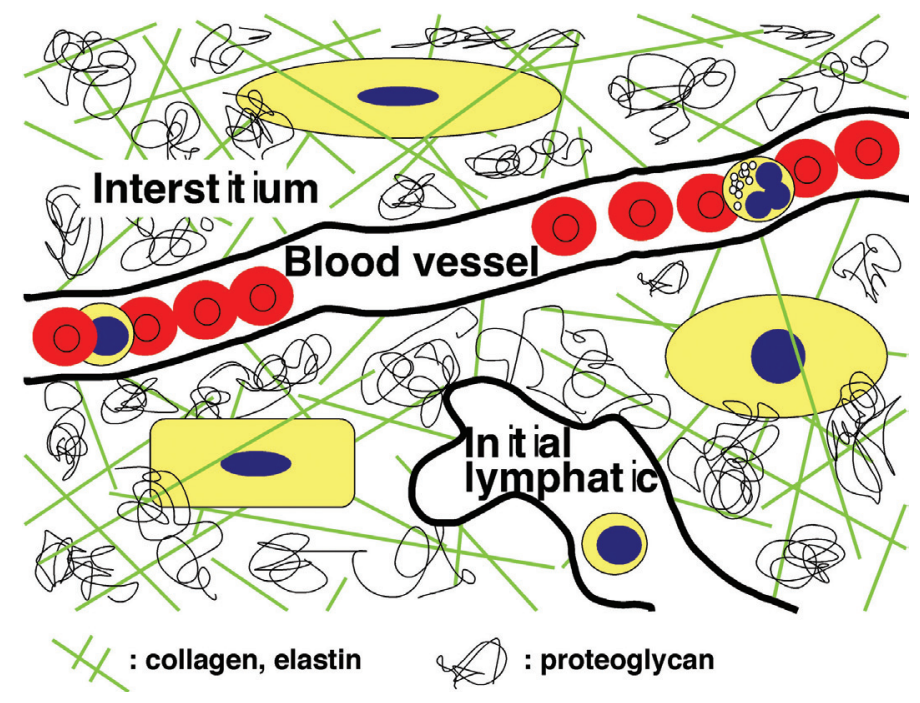

Fig. 1 Schematic representation of interstitium.

(endothelial microvalves) (Fig. 2A). Moreover, bundles of collagen fibers called anchoring filaments, which radiate to surrounding tissues, are bound to endothelial cells. Anchoring filaments are considered to prevent collapse of the initial lymphatics and even dilate them in edema despite swelling of the tissue and to contribute to drainage of tissue fluid. ${ }^{17)}$

In the skin, since the hydrostatic pressure of tissue fluid is shown to be negative, and the pressure in the initial lymphatics to be positive, some driving force is necessary for lymph to be produced against this pressure gradient. The production of lymph is known to be increased by mechanical stimuli such as arterial pulsation, vibration, and massage. ${ }^{18)}$ Moreover, it is promoted by massage when the tissue is edematous. In the light of these facts, tissue fluid is considered to be taken up due to the pumping effect created in the initial lymphatics by periodic external mechanical stimuli ${ }^{19)}$ (Fig. 2A).

\section{(3) Mechanisms of lymph transport}

The collecting lymphatics have valves in their lumens (lymphatic intraluminal valves) and contain smooth muscle in their walls. A segment of the collecting lymphatic between 2 valves upstream and downstream is called a lymphangion as a functional unit. ${ }^{16)}$ The permeability of the wall of the collecting lymphatic is relatively high to water and low-molecular-weight materials but low to macromolecular materials such as protein. ${ }^{20)}$

In the collecting lymphatics, lymph is driven by 2 mechanisms. One is called passive transport dependent on external forces exerted to the lymph vessels, and the other is active transport implemented by rhythmic spontaneous contraction of the smooth muscle in the lymph vessel wall. ${ }^{21)}$ Lymph is driven downstream if compression or contraction occurs in a lymphagion, and its regurgitation is prevented by the valves in the lumen when the lymphangion is dilated or relaxed. Thus, a pumping action is produced in each lymphangion due to repetition of compression and expansion of the tissue or contraction and relaxation of the smooth muscle in the lymph vessel wall, resulting in a unidirectional flow in the collecting lymphatics (Fig. 2B). External forces of massage, joint movements, arterial pulsation, etc. are known to induce this passive transport. Passive transport also includes movements of lymph due to gravity as in the head and neck region. In relation to active transport, the basic tension and force and frequency of spontaneous contraction of the smooth muscle of the lymph vessel wall are regulated neurally, humorally, and locally. ${ }^{22}$

\section{(4) Integration of lymph production and transport}

Lymph stagnates in peripheral regions if its production is increased, but the pumping activity of the collecting lymphatics does not increase proportionally. On the other hand, the lymph flow cannot be increased by strengthening the pumping activity of the collecting lymphatics alone if the lymph production is not increased. Thus, the lymph production and transport must be harmonized to efficiently maintain the lymph flow. Here, the stimuli that promote passive transport mentioned above all contrib- 

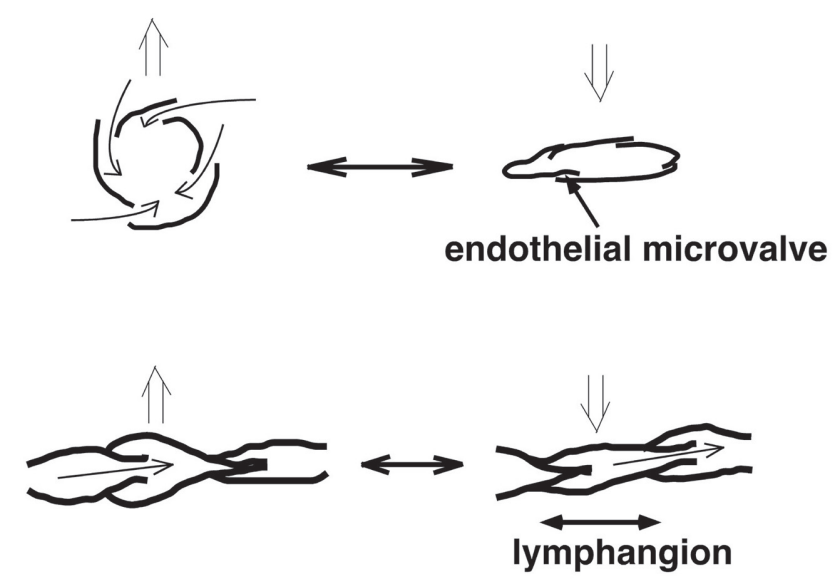

- $\forall$ : external force $\longrightarrow$ : fluid movement

Fig. 2 Effects of physical stimulation on pump function that causes lymph formation (A) and lymph transport (B).

A: Initial lymphatic (cross section)

B: Collecting lymphatic (longitudinal section)

(C)2000 Shinshu Igakukai. All Rights Reserved. Ikomi, F. Colloidal Transport through the Lymphatic System: Lymph Circulation and Drug Targeting.The Shinshu Medical Journal 2000; 48: 305-318. (in Japanese) ${ }^{24)}$

ute to the lymph production as well. In active transport, however, stretching of the wall is known to enhance the force of spontaneous contraction, and the lymph transport is increased in proportion to the lymph production by a mechanism similar to Starling's law of the heart. ${ }^{21}$ )

To dynamically evaluate the relationship between the lymph production and lymph transport, a method similar to the analytical method using the venous return curve and cardiac output curve in blood circulation by Guyton can be applied. ${ }^{23)}$ Specifically, the pressureflow relationship is evaluated by making antegrade and retrograde cannulation at a point in a lymph vessel, and the relationship between the outflow pressure and flow (lymph formation curve) and the relationship between the infusion pressure and flow (lymph transport curve) are plotted on the same coordinates (Fig. 3A). If the pressure in the lymph vessel is high, the lymph production from upstream is suppressed, and the lymph transport downstream is promoted. On the other hand, if the pressure is low, the situation is reversed. Therefore, the amount of the flow agrees at the point of the pressure shown as the intersection of the these 2 curves (equilibrium point) ${ }^{23)}$ (Fig. 3A). For example, when limb movements are increased, the pumping activity of both the initial and collecting lymphatics is enhanced, the lymph formation curve shifts upward, the lymph transport curve shifts to the left, and equilibrium is reached at a new point of intersection (unpublished data). By using this analytical method, the equilibrium point moves primarily upward, and the mechanism of the increase in the flow without much change in the pressure can be understood ${ }^{24)}$ (Fig. 3B).

To comprehensively understand the lymph kinetics, it is necessary to exhaustively collect information including the pressure and flow distributions, permeability of various parts, and composition of the lymph produced in the lymphatic system of the body by an approach that may be called system lymphology. This approach makes simulation and more detailed interpretation of the effects of local changes in the lymph vessel function on the entire lymph dynamics, effects of lymph vessel obstruction due to lymph node dissection, etc. on lymph dynamics upstream and downstream, and changes in lymph dynamics caused by generalized disorders including heart failure and liver cirrhosis possible. Such integrated interpretation of lymph dynamics is a future issue.

\section{Flow at Lymph Nodes}

\section{(1) Mechanism of lymph concentration}

Lymph is gradually concentrated, and its protein 

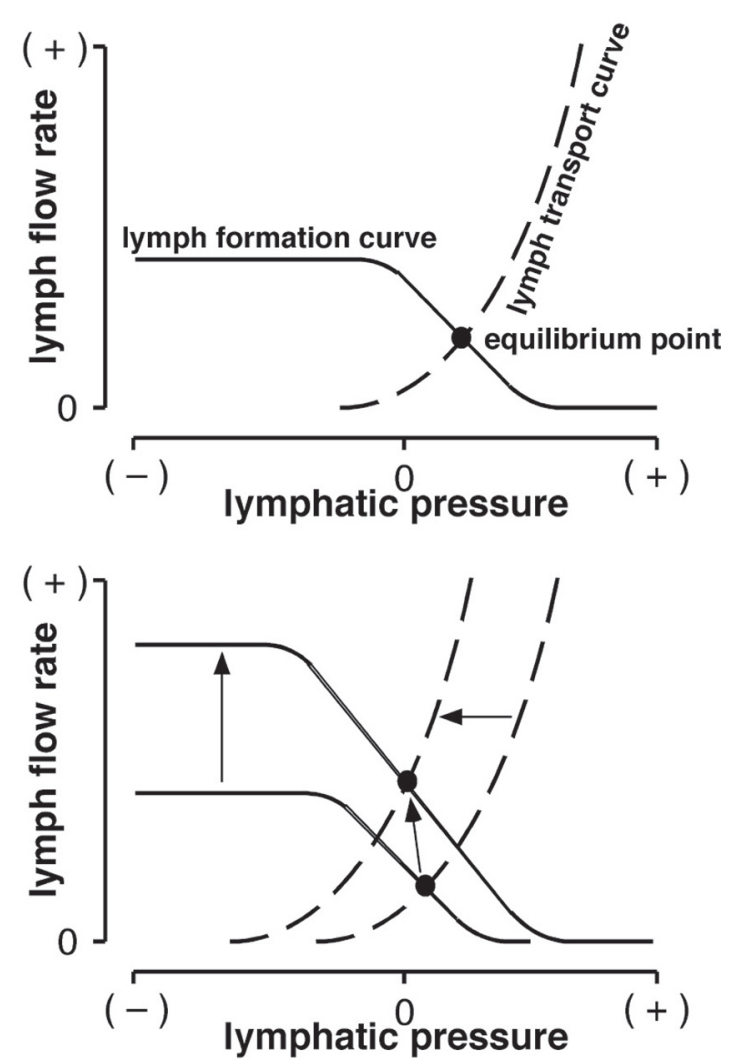

Fig. 3 Lymph dynamic analysis. The equilibrium point, which is the crossing point of a lymph formation curve (broken line) and a lymph transport curve (solid line), determines lymphatic pressure and lymph flow rate simultaneously (A). The equilibrium point is changed by shifting of the two curves (B).

C2000 Shinshu Igakukai. All Rights Reserved. Ikomi, F. Colloidal Transport through the Lymphatic System: Lymph Circulation and Drug Targeting. The Shinshu Medical Journal 2000; 48: 305-318. (in Japanese) $^{24)}$

concentration increases, as it flows downstream along the lymph vessels. ${ }^{7)}$ Concentration of lymph occurs not only in the lymph vessels but has been reported to be more notable in the lymph nodes. In the 1980s, Guyton et al. evaluated the relationship of the colloid osmotic pressure and hydrostatic pressure between plasma and lymph in detail using perfused samples of dog popliteal lymph node. The results strongly suggested: (1) Water moves, but protein does not, between blood and lymph in the lymph node, and (2) the movement of water between blood and lymph is conducted by a mechanism similar to the mechanism of water movement between the capillary and tissue space according to the Starling's hypothesis. ${ }^{7)}$ The observation that protein is concentrated as lymph travels through the lymphatic system in a physiologic state is in agreement with the above discussion. In the transport of materials, 2 factors, i.e., the concentration and flow rate, must be considered. However, from the viewpoint of recovery of plasma protein into the circulating blood, the efficiency of transport is sufficiently improved even when the loss due to the increase in the viscosity associated with concentration is taken into consideration. ${ }^{25)}$

\section{(2) Cell dynamics in lymph nodes}

Lymphocytes in the circulating blood migrate extravascularly through the HEV of the lymph nodes and return to blood via efferent lymph vessels. This release of lymphocytes from the efferent lymph vessels is known to be affected by adrenaline, histamine, serotonin, corticosteroids, and prostaglandins. ${ }^{26)}$ Recently, stimulation by sphingosine-1-phosphate (S1P) receptors has been shown to be essential to the release of lymphocytes from the lymph nodes to the efferent lymph vessels. ${ }^{27)}$ In classic experiments in which a lymph vessel was perfused with diluted plasma components, and lymphocytes in the collected lymph were counted, the possibility that lipid mediators including S1P and other physiologically active materials contained in trace amounts in the lymph affected the results cannot be excluded.

Recently, the advent of the multiphoton microscope has made possible in vivo visualization of the state of relatively deep tissues. Studies using this microscope have also gradually clarified the cell dynamics in lymph nodes. ${ }^{28,29)}$ Furthermore, analysis of the relationship between the distribution of chemokines and movements of lymphocytes is also advancing. ${ }^{11)}$ However, how the lymph flow is involved in the dynamics of these cells remains mostly unknown.

\section{(3) Contraction and relaxation of lymph nodes}

The smooth muscle present in the capsule and trabeculae of the lymph node is known to contract and relax under drug or neural stimulation. ${ }^{30)}$ As examples, Fig. 4 shows typical responses of resected specimens of dog and monkey axillary, tracheobronchial, mesenteric, and inguinal lymph nodes to adrenaline, histamine, serotonin, corticosteroids, and prostaglandins. Although the significance of this contraction and relaxation is unclear, it may be (1) regulation of the resistance to the lymph flow, (2) promotion of the pumping function for lymph and lymphocytes, and (3) regulation of the volume of the lymph node. See another review for details of contraction/relaxation responses 


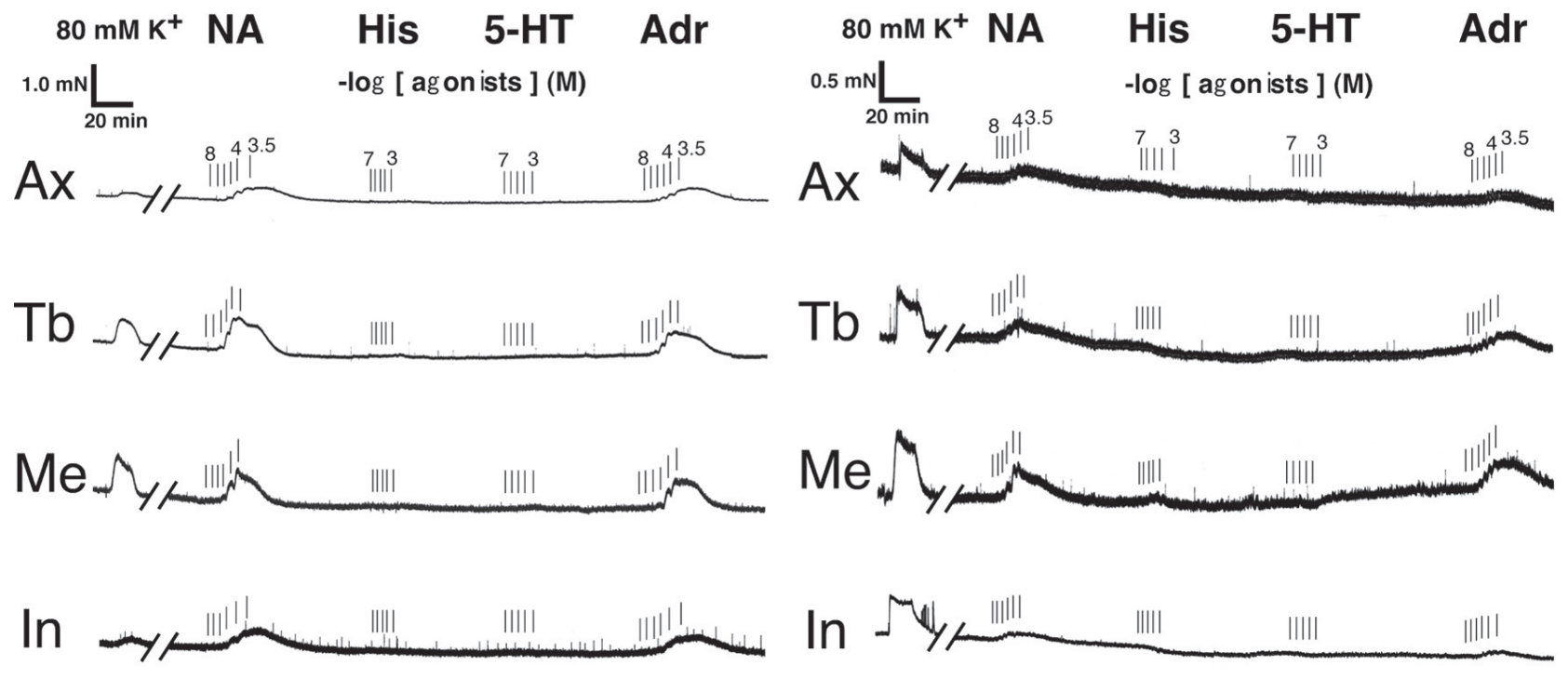

Fig. 4 Representative recordings of the effects of vasoactive amines in isolated canine and monkey lymph nodes. NA: noradrenaline; His: histamine; 5-HT: 5-hydroxytryptamine; Adr: adrenaline; Ax: axillary lymph node; Tb: tracheobronchial lymph node; Me: mesenteric lymph node; In: inguinal lymph node

of the smooth muscle in the lymph node capsule and trabeculae. ${ }^{31)}$

\section{Transport of Particles and Its Application TO THE DDS}

\section{(1) Uptake of particles into lymph vessels}

Folds of endothelial cells, which constitute endothelial microvalves in the initial lymphatics, create gaps with a width reaching several micrometers, and particulate materials injected into the tissue space are taken up into lymph vessels via these gaps (Fig. 5A). Particles in tissue spaces are known to be taken up into lymph vessels directly (extracellular transport) or indirectly by macrophages, etc., which phagocytose them and enter the lymph vessels (intracellular transport). ${ }^{32)}$ When these two modes of particle uptake are compared using fluorocarbon emulsion, extracellular transport, or direct uptake, is predominant. Also, extracellular transport is most notable immediately after particle injection and decreases gradually thereafter, but intracellular transport is minimally observed immediately after the injection but continues even after 1 week, showing a difference in time-dependence. ${ }^{32)}$ In addition, the particle concentration in lymph and lymph flow increased 10-100 times by massaging the injection site. ${ }^{19,32)}$ These observations sug- gest that the uptake of particles by the lymphatic system increases markedly by an enhancement of the pumping function of the initial lymphatics.

The uptake of particles by the lymph vessels is also known to be affected by the diameter and surface properties of the particles. Even particles with a diameter of several micrometers are shown to be transferred into the lymph vessels through tissue spaces. Also, the uptake of particles by the lymph vessels increases as the particle size is smaller. However, the uptake of fluorocarbon emulsion consisting of particles with a similar size has been reported to change with the surfactant covering the particles. ${ }^{33)}$ Therefore, at least, the size and surface properties of the particles are considered to affect their uptake of particles by the initial lymphatics.

\section{(2) Accumulation of particles in the lymph nodes}

The lymph node has a filter function for foreign bodies, and it accumulates and disposes of the captured foreign bodies in it. Findings concerning the particlesize-dependence of the passage of particles through the lymph node, which corresponds to the filter size, have been accumulated recently. When the passage of microspheres through the rabbit popliteal lymph node was measured, few particles $2 \mathrm{~mm}$ or greater in diameter passed through the lymph node, but the passage 


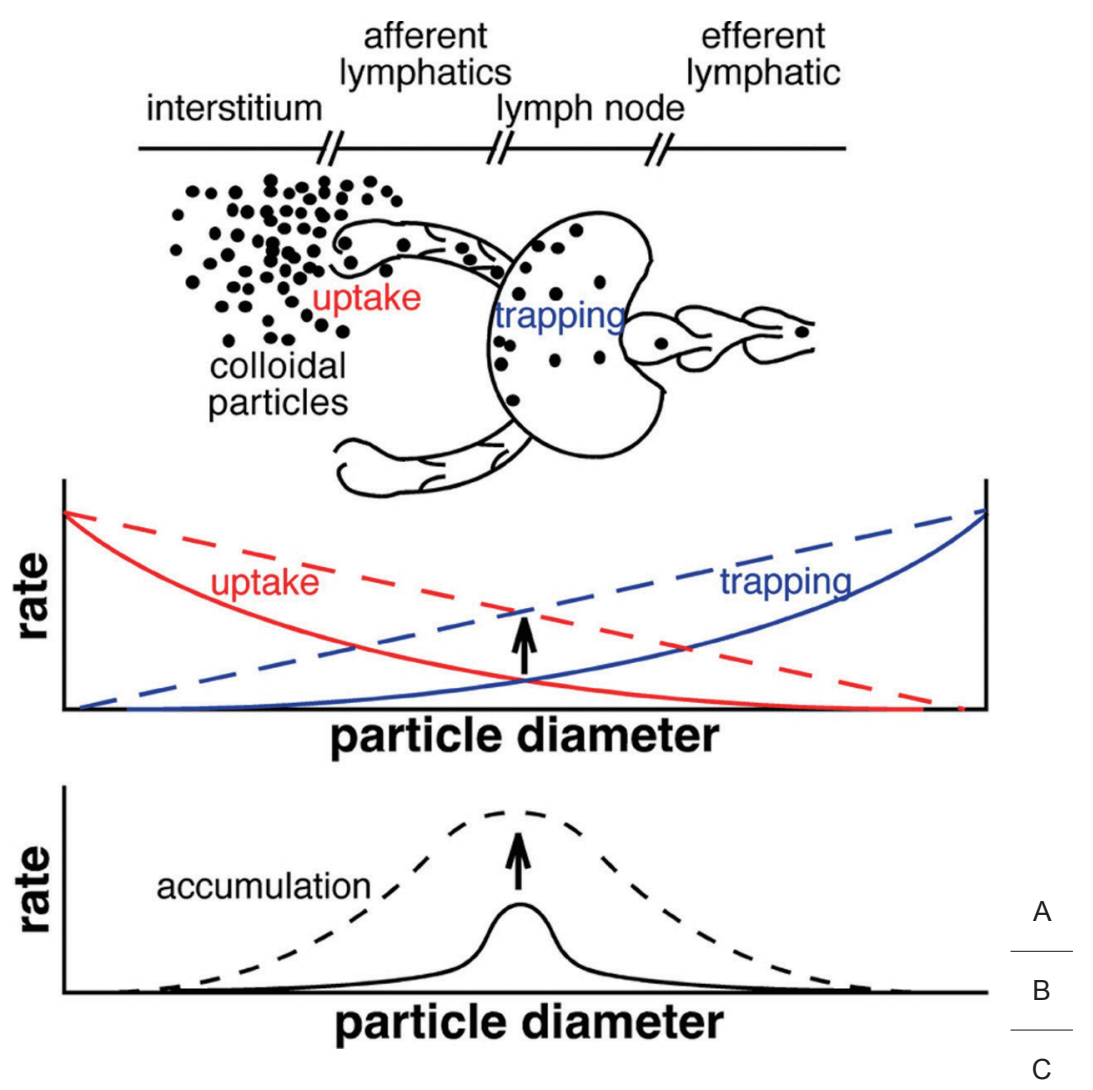

Fig. 5 Schematic representation of colloidal movement from interstitial space to lymph node (A), uptake rate (red lines) and trapping rate (blue lines) (B), and accumulation rate $(\mathbf{C})$. There may be an appropriate particle diameter for maximal accumulation in the lymph node. To increase the accumulation rate, it may be important to increase uptake rate and trapping rate (broken lines).

of smaller particles increased with decreases in their diameter in a hyperbolic manner. Along with the size barrier, the passage of lymphocytes with the same size was found to increase significantly by depleting macrophages in the lymph node with liposomes containing $\mathrm{CL}_{2} \mathrm{MBP}$ (unpublished data).

To concentrate a maximum number of particles in the lymph nodes by a DDS, it is necessary to increase at least either the uptake of particles from the tissue spaces or the capture of particles in the lymph nodes (Fig. 5B and 5C). Here, factors that affect the capture of particles at the lymph node are considered to include biological factors such as the distribution and activity of phagocytes and the expression of adhesive molecules as well as physical factors such as the diameter and surface properties of the particles, but the clarification of these problems is left to future studies.

\section{(3) Search for sentinel lymph nodes}

As a DDS from the tissue space to the lymph node, the search for sentinel lymph nodes using radioactive colloid is conducted clinically at present. ${ }^{4,34)}$ We are engaging in the development of a method for the search of sentinel lymph nodes using ultrasound diagnostic devices. ${ }^{35)}$ Here, Figure 6 shows the results of a preliminary study in which a lymph node was examined in the Doppler mode by injecting a contrast agent into a prenodal lymph vessel. If it becomes possible to detect an ultrasound contrast agent injected into the tissue space over a long time in lymph nodes, the technique is expected to be extremely useful for the diagnosis. 


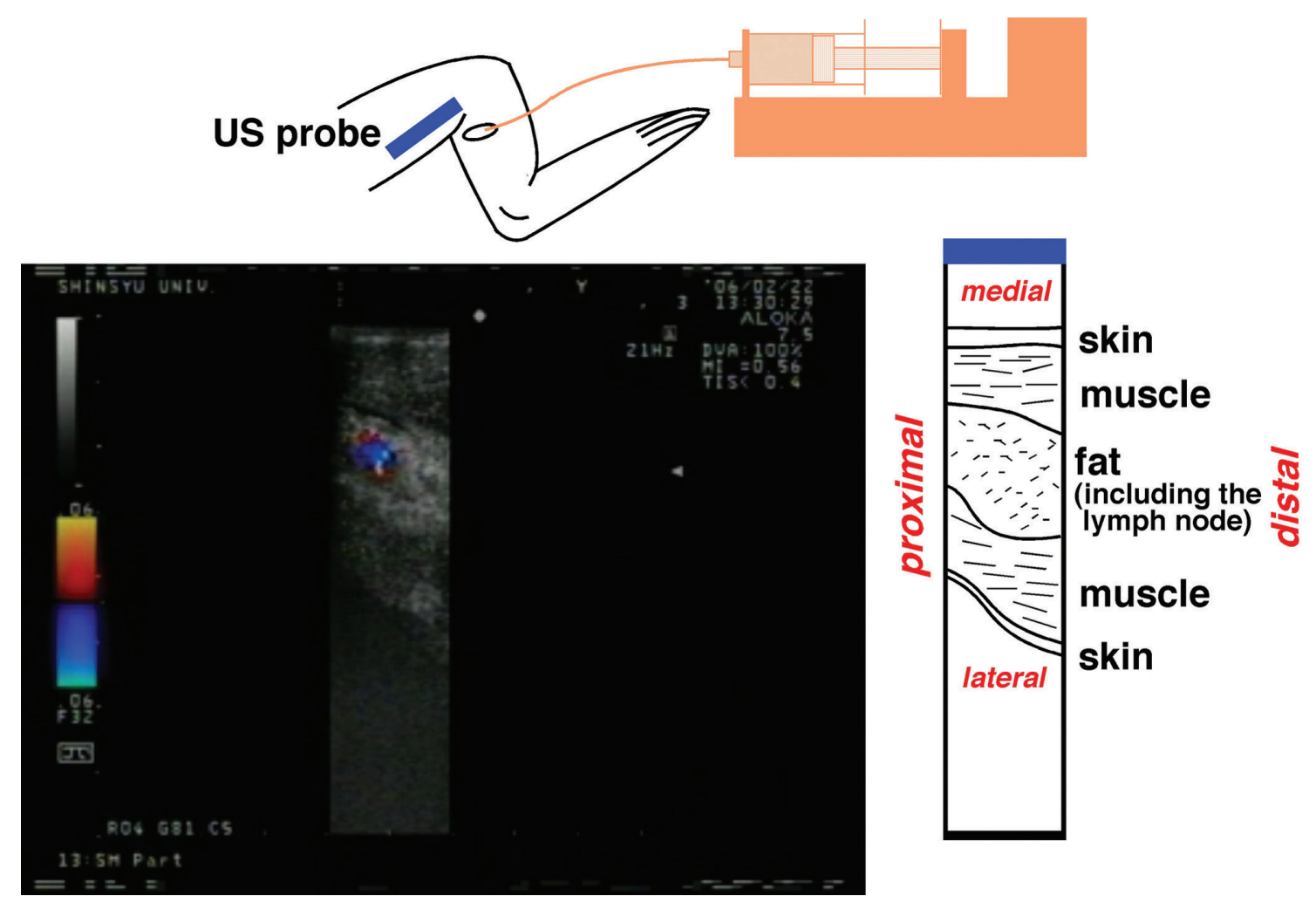

Fig. 6 Color Doppler image of the rabbit popliteal lymph node. Ultrasonographic (US) contrast medium was injected into one of the popliteal afferent lymphatics.

(C)2000 Shinshu Igakukai. All Rights Reserved. Ikomi F, Nagai T, Suzuki S, et al. Functional insights for decision and regulation of lymphatic metastasis of carcinoma cells -Development of lymph circulation-related DDS-. Lymphology 2007; 30: 13-16. ${ }^{35)}$

\section{Biology OF THE LyMPH FLOW AND LyMPHATIC SySTEM}

\section{(1) Regulation of the lymph vessel smooth muscle}

An effect of the lymph flow is shear stress on lymph vessel endothelial cells. The flow-dependent NO production and secretion have been noted in the lymphatic system as well as in the blood vessel system. ${ }^{36)}$ In an experiment using the dog thoracic duct, flow-dependent NO production and secretion were suppressed by L-arginine, which is a substrate of NO production, and NG-nitroL-arginine, which is an inhibitor of NO synthesis. In this experiment, also, no flow-dependent prostaglandin production or secretion was observed. ${ }^{36}$ )

\section{(2) Regulation of lymph vessel endothelial cells}

Shear stress applied to lymph vessel endothelial cells not only induces flow-dependent NO secretion as observed above, but we have recently demonstrated that it also enhances the gene and protein expression of endothelial constitutive NO synthase (ecNOS), an NO synthesizing enzyme and a rete-controlling enzyme in this reaction. ${ }^{37)}$ We also confirmed that the release of $\mathrm{Ca}^{2+}$ from the $\mathrm{Ca}^{2+}$ store site and activation of the $\mathrm{Ca}^{2+}{ }_{-}$ dependent $\mathrm{K}^{+}$channel are involved in this action. ${ }^{37)}$

\section{(3) Role in the lymphangiogenesis}

When the initial lymphatics are damaged, the flow of lymph that has leaked from their severed ends in the tissue space has been shown to play an important role in the repair of lymph vessels. ${ }^{38)}$ We have also obtained similar results using the rabbit collecting lymphatics. ${ }^{39)}$ When the rabbit popliteal lymph node is excised, the collecting lymphatics recanalize, and collaterals develop, at the site of excised lymph node in a course of a few weeks. However, the recanalization of the collecting lymphatics is prevented when the lymph pooled at the site of excised lymph node is drained early after surgery. In addition, the importance of lymphangiogenic factors has been suggested. ${ }^{40)}$ Details of the mechanism of the formation and 
recanalization of the collecting lymphatics, e.g., whether the physical effect of the lymph flow in the tissue space or growth factors or precursor cells of endothelial cells, etc. in lymph are important, await future clarification.

\section{Closing Remarks}

As mentioned above, the lymph vessels are considered to be a network of pumps covering the entire body. Lymph is produced and transported by this pumping effect. The composition of lymph changes during its transport. Lymph contains various physiologically active materials and immune cells as well as water, electrolytes, and plasma proteins. Knowledge of lymph dynamics is necessary to understand their transport. Moreover, knowledge about lymph dynamics is considered to contribute to the control of immune functions and the designing of DDS for the diagnosis and treatment of lymph node metastasis of tumors. I hope that this article provides a viewpoint from the lymph dynamics to many colleagues engaged in angiology.

\section{Acknowledgments}

The ultrasound contrast agent and liposomes containing $\mathrm{CL}_{2} \mathrm{MBP}$ were provided by Professor Koji Abe, Section of Macromolecule Processing and Disintegration, Department of Functional Macromolecules, Shinshu University Faculty of Textile Science and Technology. Cooperation was obtained from Messrs. Akira Teramoto and Takayuki Yanagawa of the same section in preparing these drugs.

Part of this research was subsidized by a Grand-inAid for Scientific Research by the Japan Society for the Promotion of Science (15500315, 19500384, 19209044, 22249052).

Part of this study was carried out in the Project for the Research and Development of Intelligent Surgical Instruments.

\section{REFERENCES}

1) Calnan JS, Pflug JJ, Reis ND, et al. Lymphatic pressures and the flow of lymph. Br J Plast Surg 1970; 23: 305-17. [Medline] [CrossRef]

2) Adair TH, Guyton AC: Introduction to the lymphatic system. In: Johnston MG, ed. Experimental biology of the lymphatic circulation. 1st edition ed. The Netherlands: Elsevier Science Publishers, B. V. Amsterdam, 1985, 1-12.
3) Jussila L, Alitalo K. Vascular growth factors and lymphangiogenesis. Physiol Rev 2002; 82: 673-700. [Medline]

4) Kitagawa Y, Fujii H, Mukai M, et al. Radio-guided sentinel node detection for gastric cancer. Br J Surg 2002; 89: 604-8. [Medline] [CrossRef]

5) Morton DL, Wen DR, Wong JH, et al. Technical details of intraoperative lymphatic mapping for early stage melanoma. Arch Surg 1992; 127: 392-9. [Medline] [CrossRef]

6) Starling EH. On the Absorption of Fluids from the Connective Tissue Spaces. J Physiol (Lond) 1896; 19: 312-26. [Medline]

7) Adair TH, Guyton AC. Lymph formation and its modification in the lymphatic system. In: Johnston MG, ed. Experimental biology of the lymphatic circulation. 1st edition ed. The Netherlands: Elsevier Science Publishers, B. V. Amsterdam, 1985, 13-44.

8) Tsuzuki Y, Miyazaki J, Matsuzaki K, et al. Differential modulation in the functions of intestinal dendritic cells by long- and medium-chain fatty acids. J Gastroenterol 2006; 41: 209-16. [Medline] [CrossRef]

9) Daniel PM, Gale MM, Pratt OE. Hormones and related substances in the lymph leaving four endocrine glands-the testis, ovary, adrenal, and thyroid. Lancet 1963; 1: 1232-4. [Medline]

10) Florey HW, Gowans JL. Chapter 5 The reticuloendothelial system. The omentum. The lymphatic system. The lymphocyte. In: Florey HW, ed. General pathology. W. B. Saunders Company, Philadelphia, 1962, 128-166.

11) Miyasaka M, Tanaka T. Lymphocyte trafficking across high endothelial venules: dogmas and enigmas. Nat Rev Immunol 2004; 4: 360-70. [Medline] [CrossRef]

12) Steenbergen JM, Bohlen HG. Sodium hyperosmolarity of intestinal lymph causes arteriolar vasodilation in part mediated by EDRF. Am J Physiol 1993; 265: H323-8. [Medline]

13) Kobayashi H, Pelster B, Piiper J, et al. Significance of the Bohr effect for tissue oxygenation in a model with counter-current blood flow. Respir Physiol 1989; 76: 277-88. [Medline] [CrossRef]

14) Pratt JW, Schertel ER, Schaefer SL, et al. Acute transient coronary sinus hypertension impairs left ventricular function and induces myocardial edema. Am J Physiol 1996; 271: H834-41. [Medline]

15) Dumont AE, Clauss RH, Reed GE, et al. Lymph drainage in patients with congestive heart failure. comparison with findings in hepatic cirrhosis. N Engl J Med 1963; 269: 949-52. [Medline] [CrossRef]

16) Schmid-Schönbein GW. Microlymphatics and lymph flow. Physiol Rev 1990; 70: 987-1028. [Medline]

17) Leak LV, Burke JF. Fine structure of the lymphatic capillary and the adjoining connective tissue area. Am J Anat 1966; 118: 785-809. [Medline] [CrossRef]

18) Ikomi F, Schmid-Schönbein GW. Lymph pump mechanics in the rabbit hind leg. Am J Physiol 1996; 271 (Heart Circ Physiol, 40): H173-H183. 
19) Ikomi F, Hunt J, Hanna G, et al. Interstitial fluid, plasma protein, colloid, and leukocyte uptake into initial lymphatics. J Appl Physiol 1996; 81: 2060-7. [Medline]

20) Ono N, Mizuno R, Ohhashi T. Effective permeability of hydrophilic substances through walls of lymph vessels: roles of endothelial barrier. Am J Physiol Heart Circ Physiol 2005; 289: H1676-82. [Medline] [CrossRef]

21) Ohhashi T, Azuma T, Sakaguchi M. Active and passive mechanical characteristics of bovine mesenteric lymphatics. Am J Physiol 1980; 239: H88-95. [Medline]

22) Ohhashi T, Mizuno R, Ikomi F, et al. Current topics of physiology and pharmacology in the lymphatic system. Pharmacol Ther 2005; 105: 165-88. [Medline] [CrossRef]

23) Ikomi $F$, Ohhashi $T$. Effects of leg rotation on lymph flow and pressure in rabbit lumbar lymph circulation: in vivo experiments and graphical analysis. Clin Hemorheol Microcirc 2000; 23: 329-33. [Medline]

24) Ikomi F. Colloidal Transport through the Lymphatic System: Lymph Circulation and Drug Targeting. The Shinshu Medical Journal 2000; 48: 305-18. (in Japanese)

25) Takahashi T, Shibata M, Kamiya A. Mechanism of macromolecule concentration in collecting lymphatics in rat mesentery. Microvasc Res 1997; 54: 193-205. [Medline] [CrossRef]

26) Moore TC. Neurovascular immunology: a 25 year odyssey. Ann N Y Acad Sci 1992; 650: 30-9. [Medline] [CrossRef]

27) Mandala S, Hajdu R, Bergstrom J, et al. Alteration of lymphocyte trafficking by sphingosine-1-phosphate receptor agonists. Science 2002; 296: 346-9. [Medline] [CrossRef]

28) Bousso P, Robey E. Dynamics of CD8+ T cell priming by dendritic cells in intact lymph nodes. Nat Immunol 2003; 4: 579-85. [Medline] [CrossRef]

29) Mempel TR, Henrickson SE, Von Andrian UH. T-cell priming by dendritic cells in lymph nodes occurs in three distinct phases. Nature 2004; 427: 154-9. [Med- line] [CrossRef]

30) Florey H. Observations on the contractility of lacteals: Part II. J Physiol (Lond) 1927; 63: 1-18. [Medline]

31) Ikomi $F$, Ohhashi T. Control mechanisms of lymph circulation within lymph nodes. Kekkan 2000; 23: 55-64. (in Japanese)

32) Ikomi F, Hanna GK, Schmid-Schönbein GW. Mechanism of colloidal particle uptake into the lymphatic system: basic study with percutaneous lymphography. Radiology 1995; 196: 107-13. [Medline]

33) Ikomi F, Hanna GK, Schmid-Schönbein GW. Sizeand surface-dependent uptake of colloid particles into the lymphatic system. Lymphology 1999; 32: 90-102. [Medline]

34) Ikomi F, Mizuno R, Kawai Y, et al. Sentinel lymph node concept; from lymph dynamics points of view. Clinical Surgery 2004; 59: 535-43. (in Japanese)

35) Ikomi F, Nagai T, Suzuki S, et al. Functional insights for decision and regulation of lymphatic metastasis of carcinoma cells -Development of lymph circulationrelated DDS-. Lymphology 2007; 30: 13-6. (in Japanese)

36) Tsunemoto H, Ikomi F, Ohhashi T. Flow-mediated release of nitric oxide from lymphatic endothelial cells of pressurized canine thoracic duct. Jpn J Physiol 2003; 53: 157-63. [Medline] [CrossRef]

37) Kawai Y, Yokoyama Y, Kaidoh M, et al. Shear stressinduced ATP-mediated endothelial constitutive nitric oxide synthase expression in human lymphatic endothelial cells. Am J Physiol, Cell Physiol 2010; 298: C647-55. [Medline] [CrossRef]

38) Boardman KC, Swartz MA. Interstitial flow as a guide for lymphangiogenesis. Circ Res 2003; 92: 801-8. [Medline] [CrossRef]

39) Ikomi F, Yokoyama Y, Ogiwara N, et al. Recanalization of the collecting lymphatics in rabbit hind leg. Microcirculation 2006; 13: 365-76. [Medline] [CrossRef]

40) Ikomi F, Kawai Y, Nakayama J, et al. Critical roles of VEGF-C-VEGF receptor 3 in reconnection of the collecting lymph vessels in mice. Microcirculation 2008; 15: 591-603. [Medline] [CrossRef] 